\title{
Effects of Sildenafil on Cerebrovascular Reactivity in Patients with Becker Muscular Dystrophy
}

\author{
Ulrich Lindberg $^{1,2}$ • Nanna Witting ${ }^{3}$ - Stine Lundgaard Jørgensen ${ }^{2}$. John Vissing ${ }^{3}$ • \\ Egill Rostrup $^{1} \cdot$ Henrik Bo Wiberg Larsson ${ }^{1,2}$ - Christina Kruuse ${ }^{2,4}$
}

Published online: 2 August 2016

(C) The American Society for Experimental NeuroTherapeutics, Inc. 2016
Sildenafil increased the event-related sensory and visual BOLD response compared with placebo $(p<0.01)$. However, sildenafil did not alter CBF, measured by MR phase contrast mapping, or the arterial diameter of the middle cerebral artery, measured by MR angiography. We conclude that nNOS may play a role in event-related neurovascular responses. Further studies in patients with BMD may help clarify the roles of dystrophin and nNOS in neurovascular coupling in general, and in patients with BMD in particular.

Keywords Becker muscular dystrophy $\cdot \mathrm{fMRI} \cdot \mathrm{EEG}$. Sildenafil $\cdot$ Neurovascular coupling

\section{Introduction}

Patients with Duchenne muscular dystrophy (DMD) and Becker muscular dystrophy (BMD) carry mutations in the dystrophin gene on the $\mathrm{X}$ chromosome that cause progressive muscle wasting, heart failure, and weakness that is generally more severe in DMD than in BMD. In addition to muscle symptoms, some patients also suffer from cognitive impairment [1-5].

Absent or reduced dystrophin expression in the skeletal muscles causes dysfunction of the membrane-bound dystrophin-associated glycoprotein complex (DGC) [6, 7]. In the DGC, dystrophin anchors neuronal nitric oxide synthase (nNOS) $[8,9]$, important for local production of nitric oxide (NO), in addition to NO produced from endothelial cells. NO stimulates cyclic guanosine monophosphate (cGMP) production, essential in smooth muscle cell relaxation [10], glutamate signaling [11], and mitochondrial function [12]. When the dystrophin protein is absent or truncated, the resulting loss of nNOS and reduced NO production causes 
"functional ischemia" owing to impaired vasodilatation during muscle exercise [13-15].

The occurrence of cognitive impairment in patients with DMD and BMD suggests a role for dystrophin in brain function [4, 5, 16-18]. Neurons, glia, and the microvasculature of the brain have a DGC-like complex, corresponding to the muscle DGC, that also anchors nNOS [19]; nevertheless, the role of dystrophin in the brain is not well established.

An altered cerebrovascular response might mimic the responses in skeletal muscles [14], but the effects of modulating the NO-cGMP cascade in the brains of patients with DMD or BMD are unknown. To investigate this, sildenafil, a selective inhibitor of phosphodiesterase type 5 (PDE5), was chosen as a tool for modulating NO-cGMP signaling in patients with BMD. Sildenafil is used to treat erectile dysfunction and pulmonary hypertension, and works by augmenting endogenous cGMP levels, thus promoting a normal NO response. Recently sildenafil/PDE5 administration was shown to reduce skeletal muscle and cardiac dysfunction and to ameliorate dystrophy in a mouse model of DMD [20-22], although no positive effect was found on skeletal or cardiac muscle function and brachial arterial forearm flow in 17 patients with BMD [23] or 15 patients with BMD/DMD [24]. In healthy subjects, including males and females, sildenafil does not change the cerebral blood flow (CBF) after a single oral dose of $100 \mathrm{mg}$ measured by single photon emission computer tomography and transcranial Doppler
[25], but it may do so in patients with dysfunctional NO signaling [26], such as patients with BMD.

We hypothesized that the altered vascular response observed in the muscles of patients with BMD is also present in the cerebral circulation, with a similar cerebral "functional ischemia" and a relative reduction of baseline or, more likely, activity-induced CBF. We investigated whether patients affected by BMD display an altered cerebrovascular response after 4 weeks of treatment with sildenafil or placebo using blood oxygen level-dependent (BOLD) functional magnetic resonance imaging (fMRI); task-based and hypercapnia challenges; perfusion; and angiography. The investigation of patients with BMD could be a relevant model for the study of the nNOS-cGMP signaling system in the brain. This would be important not only for patients with BMD, but also for expanding our understanding of $\mathrm{CBF}$ regulation in general.

\section{Materials and Methods}

\section{Subjects}

Seventeen patients with BMD (mean age 38.5 years; range 25-62 years) were included (Fig. 1, Table 1). Genetic testing and demonstration by Western blotting of deficient muscle dystrophin expression were used to confirm the diagnoses. Fourteen of the patients had deletion mutations between exons
Fig. 1 Flow chart showing patient inclusion. Twelve patients completed all 3 scans

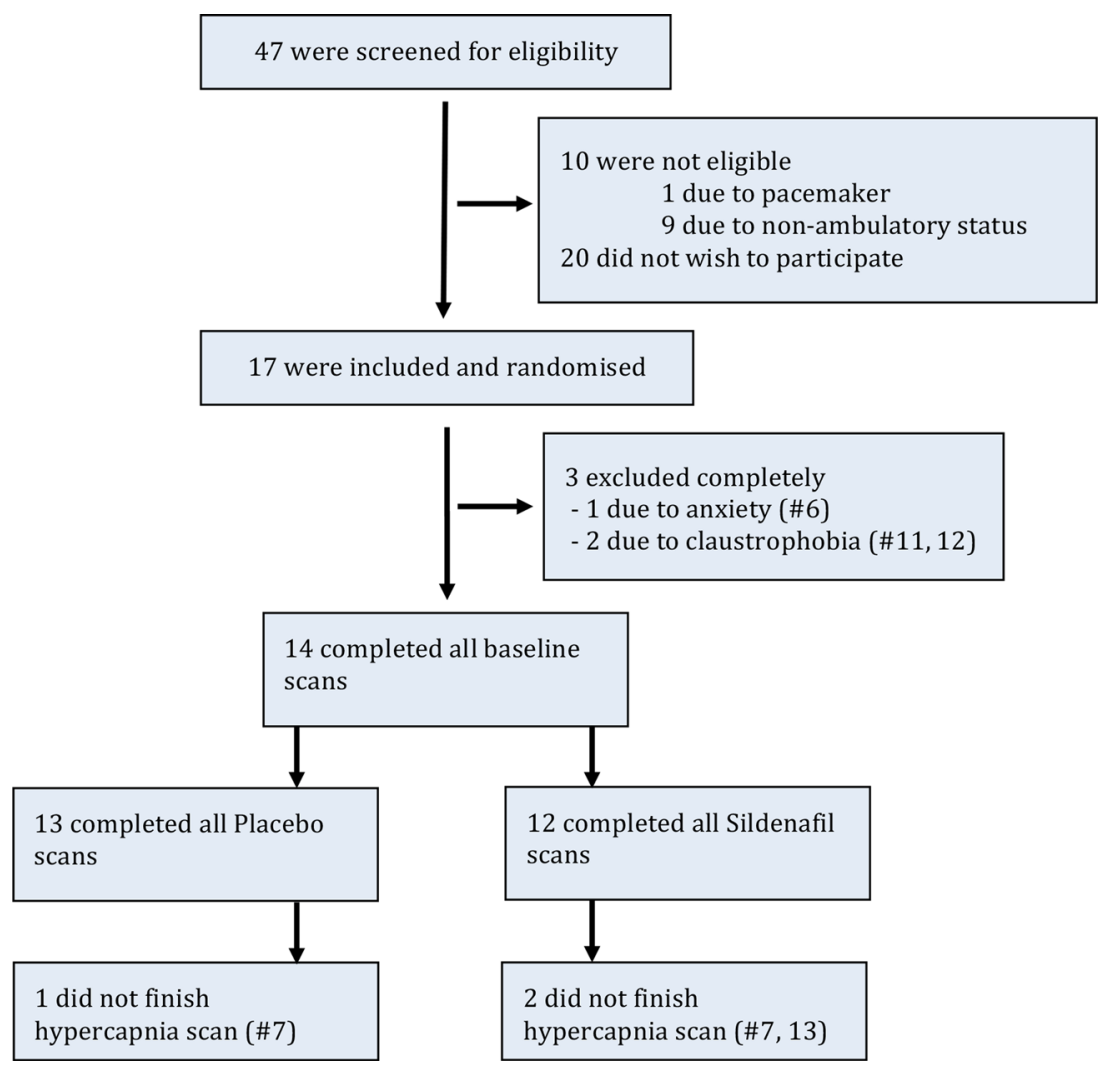


Table 1 Baseline characteristics of the patients with Becker muscular dystrophy in this study

\begin{tabular}{lllllllllllllllll}
\hline Patient & 1 & 2 & 3 & 4 & 5 & 7 & 8 & 9 & 10 & 11 & 12 & 13 & 14 & 15 & 16 & 17 \\
\hline Age (years) & 30 & 41 & 28 & 53 & 31 & 25 & 39 & 33 & 41 & 39 & 62 & 57 & 27 & 39 & 31 & 25 \\
Handedness & $\mathrm{L}$ & $\mathrm{R}$ & $\mathrm{R}$ & $\mathrm{R}$ & $\mathrm{R}$ & $\mathrm{R}$ & $\mathrm{R}$ & $\mathrm{R}$ & $\mathrm{R}$ & $\mathrm{R}$ & $\mathrm{R}$ & $\mathrm{R}$ & $\mathrm{R}$ & $\mathrm{R}$ & $\mathrm{R}$ & $\mathrm{R}$ \\
Treatment group & $\mathrm{A}$ & $\mathrm{A}$ & $\mathrm{B}$ & $\mathrm{B}$ & $\mathrm{A}$ & $\mathrm{B}$ & $\mathrm{B}$ & $\mathrm{B}$ & $\mathrm{B}$ & $\mathrm{A}$ & $\mathrm{A}$ & $\mathrm{B}$ & $\mathrm{A}$ & $\mathrm{B}$ & $\mathrm{A}$ & $\mathrm{B}$ \\
Stimulus strength (mA)* & & & & & & & & & & & & & & & \\
$\quad$ Visit 1 & 4.90 & 5.30 & 6.00 & 3.38 & 5.50 & 3.07 & 5.00 & 4.01 & 4.01 & 3.25 & 3.90 & 3.90 & 2.90 & 2.50 & 5.20 & 4.43 \\
$\quad$ Visit 2 & 4.30 & 5.00 & 5.30 & 6.00 & 5.75 & 3.53 & 4.00 & 4.01 & 3.51 & $\dagger$ & $\dagger$ & 3.50 & 2.70 & 2.44 & 4.74 & 5.01 \\
$\quad$ Visit 3 & 3.60 & 5.40 & 5.02 & 4.96 & 5.06 & 3.50 & 3.50 & 3.49 & 3.40 & $\dagger$ & $\dagger$ & 3.88 & 3.12 & 4.80 & 4.25 & 3.05 \\
\hline
\end{tabular}

$\mathrm{L}=$ left handed; $\mathrm{R}=$ right handed; $\mathrm{A}=$ active then placebo; $\mathrm{B}=$ placebo then active

*Stimulus strength refers to the applied current to the radial nerve during the electrical stimulation functional magnetic resonance task

${ }^{\dagger}$ Left study due to claustrophobia

45 and 48, which put them at risk of being deficient in the Dp140 isoform localized to the central nervous system. This has been associated with changes in $\mathrm{CBF}$ and cognitive deficits $[5,27]$. The design was a randomized double-blind, placebo-controlled, crossover study of two 4-week treatments with either placebo (lactose monohydrate oral in gelatin capsules, 3 times daily) or sildenafil $20 \mathrm{mg}$ [Revatio in gelatin capsules (Pfizer, New York, NY, USA), p.o., 3 times daily], with the treatments separated by a washout period of 2 weeks.

Patients underwent fMRI scanning 3 times: at baseline, at 4 weeks (after the end of the first treatment period), and at 10 weeks (after the end of the second treatment period) at approximately the same time of day (Fig. 2). Patients refrained from consuming caffeine-containing drinks or food for $12 \mathrm{~h}$ prior to each scan. Three patients dropped out after the first scan, 1 owing to anxiety and 2 owing to claustrophobia after completing parts of the first scan. Furthermore, 2 patients did not complete scans during hypercapnia, owing to discomfort during scanning.

Before inclusion in the study, written informed consent was obtained from all patients according to the Declaration of Helsinki. The study was approved by the Danish National Committee on Health Research Ethics (EudraCT \# 2010-024659-10) and was registered at clinicaltrials.gov (\#NCT01350154).

\section{MRI and Stimulation}

MRI was performed on a $3.0 \mathrm{~T}$ Philips Achieva scanner (Philips Medical Systems, Best, the Netherlands) using a 32-channel head coil. Anatomic and functional images were acquired to determine if sildenafil induced changes in the basal $\mathrm{CBF}$, task-induced $\mathrm{CBF}$, or middle cerebral artery (MCA) circumference. A detailed overview of the important parameters for all MRI scans is in the supplementary section (Appendix S1).

\section{BOLD imaging}

Two scans were performed using BOLD contrast imaging. For the electrical stimulation, all patients underwent 2 identical somatosensory electrical stimulation tasks, 1 outside the scanner with electroencephalography recording and 1 inside the scanner during BOLD recording. All parameters were identical for the 2 runs. Delivery of the electrical impulses was powered by a DS7A High Voltage Constant Current Electrical Stimulator (Digitimer, Welwyn Garden City, UK). The stimulation electrode was placed over the median nerve on the dominant side, as determined by the Edinburgh Handedness Scale, and the intensity was set to $80 \%$ of the motor threshold. Stimulus strength is dependent of the exact position of the stimulating electrode relative to the nerve, why it was necessary to calibrate this prior to each session. Handedness was not considered an exclusion criterion for the investigation of somatosensory, visual, or motor function. Postprocessing included mirroring of the data to allow for comparisons at the group level.

There was no significant difference in stimulation strength between the 3 scanning days. The stimulation paradigm consisted of 3 blocks of variable duration $(2,4$, and $10 \mathrm{~s})$ repeated 10 times in random order with a stimulation frequency of $5 \mathrm{~Hz}$ and an interval of $30 \mathrm{~s}$ between stimulations to allow the BOLD response to normalize. The Philips scanner
Fig. 2 The crossover study design. MRI = magnetic resonance imaging
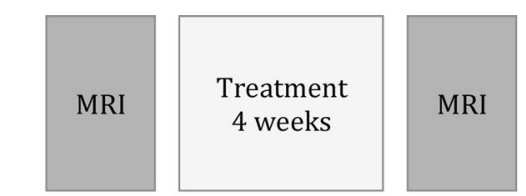

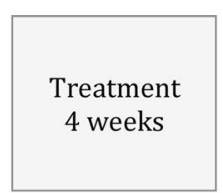

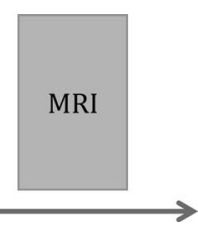


software (iViewBold) allowed for a simple BOLD correlation to verify activation of the somatosensory areas during the run.

The visual paradigm consisted of a $30 \mathrm{~s}$ on $30 \mathrm{~s}$ off flickering checkerboard reversing at $7 \mathrm{~Hz}$ with a red fixation cross in the middle. When the checkerboard stimulation was on, the patient was asked to open and close their dominant hand to create activation in the motor cortex. The movement of the hand was confirmed through the window of the operator's room.

\section{Global CBF and angiography}

A multichunk 3-dimensional (3D) inflow MRI angiogram was acquired to localize the main cerebral arteries. The global $\mathrm{CBF}$ measurement slice was then placed as orthogonally as possible to the internal carotid arteries and to the basilar artery to minimize partial volume effects and to avoid turbulent flow. The phase contrast mapping measurements were performed using phase subtraction of a flow-compensated and flow-sensitive gradient echo sequence. Angiography of the cerebral arteries was imaged [MR angiography (MRA)] using a $3 \mathrm{D}$ time-of-flight technique. Imaging was performed in the axial plane, centred on the Circle of Willis.

\section{Regional $C B F$}

Regional CBF mapping was performed using pseudocontinuous arterial spin labeling (pCASL). Imaging was performed at rest and during a block design involving inhalation of hypercapnic air (2 active blocks and 3 rest blocks of 2 min each). During the active blocks, the patient inhaled $5 \% \mathrm{CO}_{2}$ gas (20\% oxygen, $75 \%$ nitrogen) through a closed mouthpiece system that did not allow rebreathing. The resting CBF was evaluated with a single-echo pCASL sequence, and the hypercapnic air inhalation paradigm was acquired with dual-echo pCASL.

\section{Postprocessing}

An in-house Matlab (MathWorks, Natick, MA, USA) script was used to convert data into NIfTI format and to calculate the global CBF from the phase contrast mapping data. In the rare cases of aliasing due to peak velocities $>100 \mathrm{~cm} / \mathrm{s}$, the velocity maps were corrected by unwrapping using a known velocity profile as template, and global CBF measures were performed by assigning regions of interest to the feeding arteries within the imaging slap and then multiplying the vessel area with the mean velocity. CBF values were quantified by calculating the total gray and white matter volumes from the 3D T1-weighted image and then dividing the total flow by the total brain volume. All other data processing, except for MCA calculations, was performed using the FSL software package (FMRIB Software Library, Functional Magnetic
Resonance Imaging of the Brain Centre, Department of Clinical Neurology, University of Oxford, Oxford, UK).

\section{fMRI data and pCASL dual echo}

General linear modeling and group statistical comparisons [analysis of variance (ANOVA)] were performed using FEAT (FMRI Expert Analysis Tool) version 6.00, which is part of FSL. Motion correction was applied, and the motion parameters were included in the patient-specific model to minimize motion-correlated activations. Absolute head motion was low, ranging from 0.13 to $1.71 \mathrm{~mm}$. A high-pass filter with a filter cut-off at $90 \mathrm{~s}$ was applied, and the data were smoothed using a 5-mm full-width half-maximum smoothing kernel. Registration to the MNI-152 brain template was carried out through linear registration from $\mathrm{AMRI}$ to T1-weighted $3 \mathrm{D}$ using a rigid body transformation and nonlinear registration from T1-weighted 3D to MNI-152 space. For optimal estimation of peak amplitude, time to peak, and duration of the BOLD response, a set of 3 basic functions was constructed using FLOBS, an FSL tool [28], to estimate the hemodynamic response function for each voxel. The different maps were then permutation tested using RANDOMISE, an FSL tool, with 5000 permutations, including correction for multiple comparisons. The pCASL dual echo was analyzed similarly to the fMRI data using FEAT with a design matrix that included estimations of $\mathrm{CBF}$ and the BOLD response to the hypercapnia task. A comparison of the treatment groups was performed using repeated measures ANOVA.

\section{MR angiography data}

Dimensions of the MCA were assessed using the stand-alone LKEB-MRA vessel wall analysis software [29]. A stretch of the MCA was chosen that started at its origin at the internal carotid artery and finished approximately $20 \mathrm{~mm}$ downstream from that point. A 5-mm section of the $20-\mathrm{mm}$ window, which did not contain any branches, was chosen for analysis. The MCA section was placed carefully for each scan to avoid any drawing-related differences. The cross-sectional area, circumference, and diameter were calculated for each scan.

\section{Statistics}

All statistical analysis was performed using repeated measures ANOVA testing. The tests were performed using Stata (StataCorp, College Station, TX, USA) or using the FSL software for the voxel-wise image analysis. Plots for viewing were generated using FSL and Matlab. All voxel-wise statistics were corrected for multiple comparisons. The region of interest-based ANOVA and $t$ test were not corrected. 


\section{Results}

Anatomical sequences were reviewed by a neuroradiologist, who confirmed the absence of structural abnormalities in all patients.

\section{CBF}

The total blood flow to the brain as measured by the phase contrast method yielded a mean value of $53 \mathrm{ml} / 100 \mathrm{~g} / \mathrm{min}$, which is well within the expected range (Table 2). No significant treatment effect was detected. The relative regional CBF maps as determined by pCASL showed no voxel-based treatment effects.

\section{Somatosensory Stimulation}

The median nerve stimulation elicited a strong positive BOLD response for all stimulation durations in all patients, both in the contralateral primary somatosensory cortex, and as bilateral activation in the secondary somatosensory cortex. The stimulation strength was not significantly different for the 3 scans $(p=0.66)$.

Placing an anatomically defined region in the primary somatosensory cortex using the Jüelich Histological Atlas, available in the FSL toolbox [30, 31], revealed a significant increase in the BOLD amplitude with sildenafil treatment versus placebo $(p=0.0042)$. Computing voxel-wise comparisons of the whole brain using 3 basis functions for optimal modeling of the hemodynamic response function (using the FLOBS toolkit, which is part of FSL) suggested a treatment effect in areas that overlap the activated areas of the somatosensory cortex (Fig. 3). The amplitude of the BOLD response was significantly increased (uncorrected, $p<0.05$ )

Table 2 Results of the cerebral blood flow (CBF), blood oxygen leveldependent (BOLD) activation, and parameters of the hemodynamic response function from FLOBS modeling in all three conditions

\begin{tabular}{|c|c|c|c|}
\hline Measurement & Baseline & Placebo & Sildenafil \\
\hline $\mathrm{CBF}(\mathrm{ml} / 100 \mathrm{~g} / \mathrm{min})$ & $56.2 \pm 2.70$ & $52.4 \pm 2.70$ & $49.5 \pm 2.40$ \\
\hline BOLD motor $(\%)$ & $0.23 \pm 0.03$ & $0.14 \pm 0.09$ & $0.23 \pm 0.07$ \\
\hline BOLD visual $(\%)$ & $1.67 \pm 0.19$ & $1.12 \pm 0.16$ & $1.61 \pm 0.20 *$ \\
\hline $\mathrm{CO}_{2}$ reactivity $(\%)$ & $2.41 \pm 0.18$ & $2.78 \pm 0.22$ & $2.64 \pm 0.24$ \\
\hline \multicolumn{4}{|c|}{ FLOBS of BOLD somatosensory } \\
\hline Time-to-peak (s) & $7.75 \pm 1.15$ & $7.99 \pm 1.13$ & $6.57 \pm 0.80$ \\
\hline Peak (a.u.) & $0.25 \pm 0.09$ & $0.16 \pm 0.03$ & $0.38 \pm 0.08 *$ \\
\hline RMS (a.u.) & $0.04 \pm 0.004$ & $0.04 \pm 0.003$ & $0.04 \pm 0.003$ \\
\hline
\end{tabular}

Data are mean $\pm \mathrm{SD}$. The first column is from a repeated-measure analysis of variance of all three states

RMS $=$ root mean square; a.u. = arbitrary units

* Significant difference between sildenafil and placebo using a paired $t$ test with a confidence interval of $95 \%$

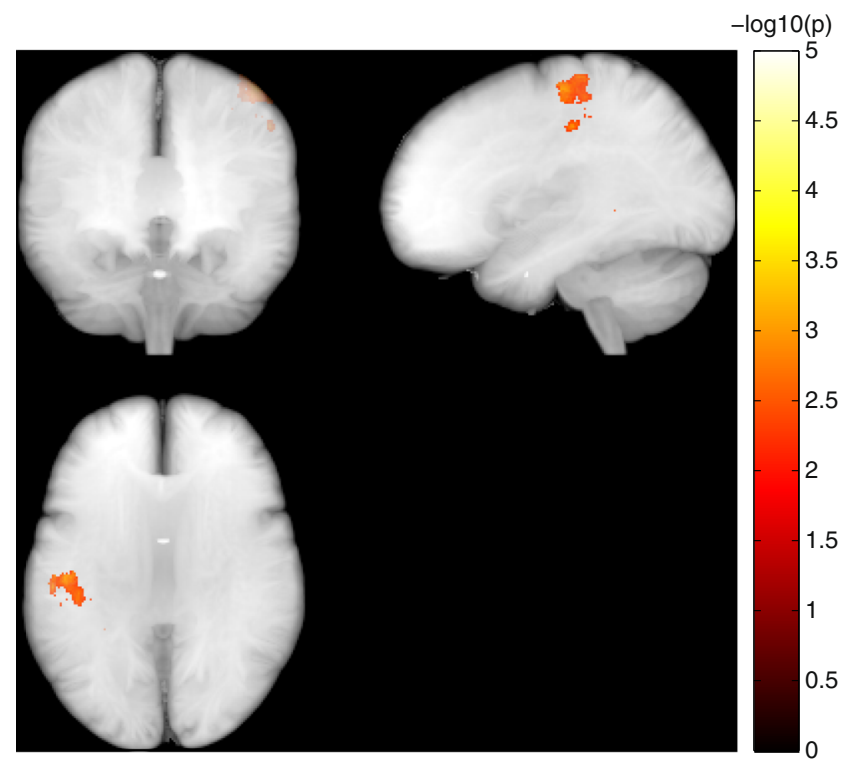

Fig. 3 Group results for the treatment effect on blood oxygen leveldependent (BOLD) response peak amplitude. Voxel-wise permutationbased tests showed significant increases in the BOLD amplitudes in the contralateral (left) primary somatosensory cortex for sildenafil treatment compared with placebo (uncorrected $p<0.05$ )

by treatment with sildenafil. Sildenafil treatment showed no effect on the full-width half-maximum duration of the BOLD response.

The electroencephalographic data acquired outside the scanner revealed no treatment effect on the root mean square value or on the N20-P26 amplitude of the somatosensoryevoked potential (Fig. S1).

\section{Visual/Motor Task fMRI}

The functional visual and motor task elicited a strong response in areas corresponding to the visual and contralateral motor cortex together with the somatosensory cortex, thalamus, and the supplementary motor area (Fig. 4). Applying a region of interest to the $\mathrm{V}_{1}$ region using the MNI-152 space Jüelich Histological Atlas [30,31], and transforming it to the native space of the BOLD scans using nonlinear registration (FNIRT, part of the FSL software), revealed a significantly increased BOLD response during sildenafil treatment compared with placebo $(p<0.03)$ (Table 2). Group voxel-based statistical analysis did not show any significant impact of treatment on the BOLD response.

\section{$\mathrm{CO}_{2}$ Reactivity}

Inhalation of hypercapnic gas $\left(5 \% \mathrm{CO}_{2}\right)$ elicited markedly $\mathrm{CBF}$ and BOLD increases globally in the brain, with a mean signal increase of $2.61 \%$ (Table 2), which is well in line with earlier findings [32]. End-tidal $\mathrm{CO}_{2}$, which was measured using a capnograph (MP150; Biopac Systems, Goleta, CA, 


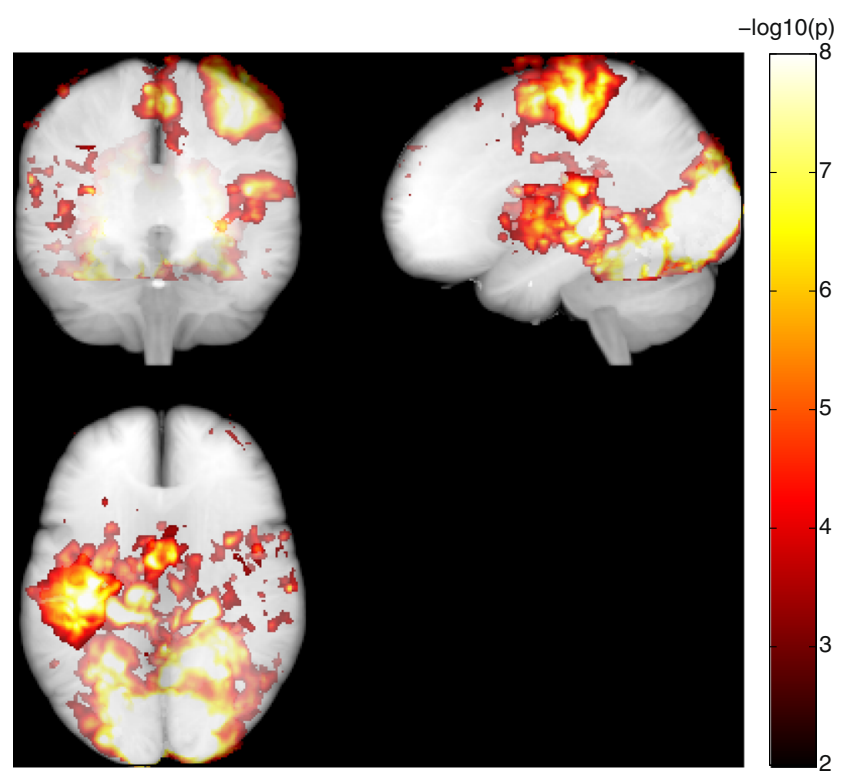

Fig. 4 Mean activation maps as projected onto a glass brain representation from visual and motor paradigms from all patients during treatment. Strong blood oxygen level-dependent responses were present in the visual cortex, contralateral motor cortex, thalamus, and supplementary motor area. The activation maps were similar at baseline and after placebo treatment

USA), was $35.7 \mathrm{mmHg}$ for the normoxic periods and $47.8 \mathrm{mmHg}$ for the hypercapnic periods, with a significant difference between the states $(p<0.001)$. A small but nonsignificant increase $(p=0.35)$ in respiration frequency from 11.6 respirations/min to 12.1 respirations/min was also seen. The mean BOLD activation maps showed that hypercapnia had a global effect (Fig. 5). Treatment had no significant impact on regional differences, as shown using a voxel-wise test for regional differences using group statistics.

\section{MCA Dimensions}

The MCA diameter was measured in both hemispheres. Treatment had no significant effects on any of the calculated parameters (Table S1).

\section{Discussion}

Four weeks of treatment with the selective PDE5 inhibitor sildenafil significantly increased cerebrovascular reactivity to somatosensory stimulation in patients with BMD. Our hypothesis that sildenafil would modulate the cerebrovascular event-related response in patients with BMD was confirmed as shown by the positive impact of sildenafil on somatosensory activation, measured by BOLD fMRI. Thus, patients with BMD are likely to have an altered stimulation-induced cGMP-NO response in the cerebrovasculature, similar to that reported in muscles. To confirm this fully, additional studies comparing responses to healthy subjects are needed.

Sildenafil treatment did not affect the basal diameters of the large cerebral arteries as measured by MRA, and CBF as measured by arterial spin labeling was unchanged, suggesting no basal effects on arterioles. The lack of a basal vascular response corresponded to the effect of sildenafil as used to treat erectile dysfunction; in that case, stimuli are required for vascular effects to occur. The unaffected somatosensoryevoked potential argues against a purely neuronal effect. Thus, given the significant change in the event-related BOLD response, it could be argued that in patients with BMD who display reduced nNOS, sildenafil most likely alters task-induced neurovascular coupling.

We also detected an increase in the visual response after sildenafil treatment compared with placebo; however, the change was small and not significant in the comparison with the baseline data.

The sensory system was investigated using an event-related design owing to the nature of the sensory stimulus, whereas the visual and motor systems were investigated using a block design. It is optimal to evaluate the hemodynamic response using an event-related design, whereas a block design is better for detecting differences in response amplitude given that the shape of the response is constant between 2 activation states. In the present study, we found that changes in the BOLD response after sildenafil were most prominent
Fig. 5 Blood oxygen leveldependent cerebrovascular reactivity $(\% / \mathrm{mmHg})$ at baseline (left) and in the placebo (middle) and sildenafil (right) groups in response to $5 \%$ hypercapnia

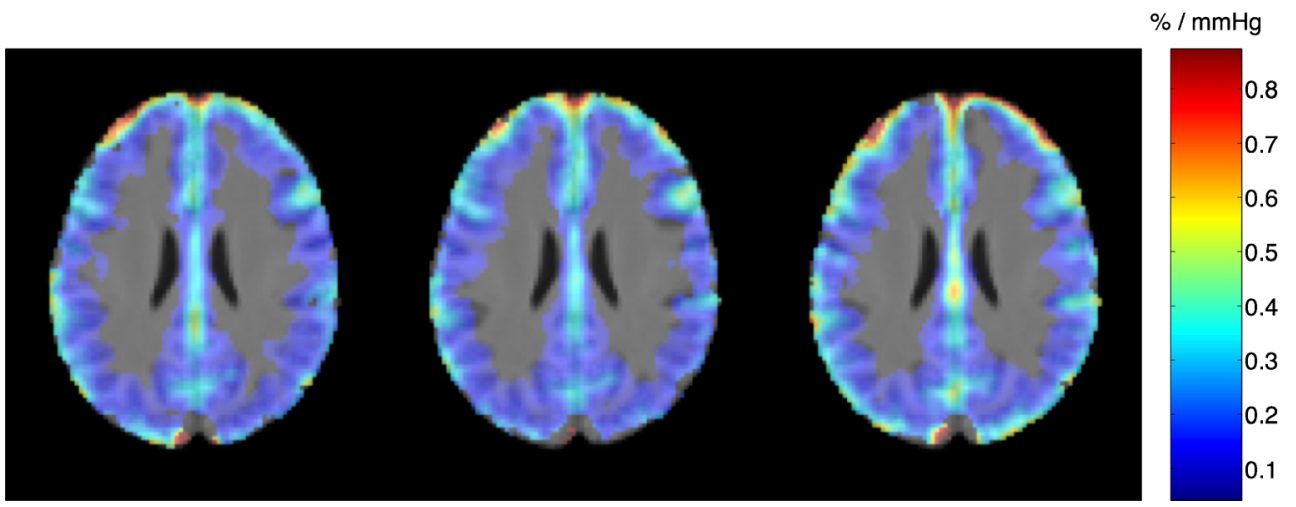


in the event-related design, which takes the variability of the hemodynamic response shape into account. This suggests that it is essential to model the response very accurately.

The impact of dystrophin gene mutations on neuronal excitability, cerebral perfusion, and CBF reactivity is only partly elucidated; in boys with DMD, positron emission tomography [33], MR spectroscopy of the brain [34], and, recently, resting state fMRI [35] suggest reduced glucose metabolism in brain tissue [33], as well as a decrease in the motor cortex synchronization of spontaneous activity in patients with DMD versus healthy controls as detected using resting state fMRI of the motor cortex [35]. Further, reduced cerebral blood flow [27] and a global reduction in cerebral gray matter [36] has been reported in boys with DMD.

The reported reduced recruitment of sufficient blood flow in patients with BMD during muscle activity responsible for relative ischemia [37] is improved by PDE5 inhibition in some studies [22, 38, 39], but not in all of them [23, 24], which may relate to variations in methods applied. Corresponding reduced brain blood flow recruitment during activation was hypothesized to be present in patients with BMD and restored by PDE5 inhibition; the current data partly support this hypothesis.

Dystrophin is present in the brain linked to nNOS signaling, neuronal excitability, and the vascular response $[17,40]$. In the brain, dystrophin localizes to neurons and glial cells in the hippocampal, cortical, and subcortical regions, as well as to the Purkinje cells of the cerebellum [41]. Full-length dystrophin is expressed mainly in neurons. A shorter isoform, the abundantly expressed Dp71, is found in perivascular astrocytes and in Bergmann glia cells. Other isoforms show specific localization, such as Dp140 in the microvasculature, Dp260 in the retina, and Dp116 in the Schwann cells of peripheral nerves [16]. The DGC-like complex and nNOS are suggested to modulate the $\gamma$-aminobutyric acid response, which, in turn, can affect the microcirculation $[17,18,40,42]$.

In healthy subjects, sildenafil does not change CBF or neuronal activity as measured by single photon emission computed tomography and by the BOLD response during visual and $\mathrm{CO}_{2}$ stimulation [25, 43]. Sildenafil may nevertheless increase the blood flow velocity in patients with supposed dysfunctional NO signaling, as measured by transcranial Doppler, with resulting beneficial effects on cerebral vascular reactivity [26]. The cerebrovascular effects of sildenafil are primarily reported in systems with dysfunctional NO signaling [26, 44] rather than in systems in which NO-cGMP signalling is normal [25, 45]. Interestingly, neuroprotective and neurorestorative effects of sildenafil are reported in animals [46-48], though such effect needs to be fully confirmed in humans.
In the current study, sildenafil significantly increased the event-related BOLD response, although the patients had no sensory deficits and showed no changes in the corresponding somatosensory-evoked potential. PDE5 inhibition only minimally affected the visual BOLD response. Both results suggest that sildenafil has mainly activity-related effects. Sildenafil did not change the arterial diameter or the $\mathrm{CBF}$, which indicates that dystrophin and NO play minor roles in basal cerebrovascular regulation. The subtle effects of sildenafil suggest that nNOS may be a less important player in the regulation of CBF, with endothelial NOS contributing more to the functionally important basal NO pool. This was recently confirmed in an animal study $[49,50]$. These findings imply that endothelial NOS and nNOS have different roles in cerebrovascular reactivity and in the brain.

The patients reported headache as a side effect of sildenafil treatment, supporting previous studies showing that PDE5 and cGMP, but not large artery dilation, are key elements in headache signaling $[25,45]$.

We thus know from its reported side effects that sildenafil is pharmacologically active at the dose used in this study. In animal studies sildenafil is reported to pass the blood-brain barrier [51], though similar is not clearly evident in humans [52]. Muscle biopsies from the patients showed reduced nNOS protein expression and a corresponding reduction in PDE5 expression [23], which could cause the patients to be less sensitive to PDE5 inhibitors.

Only 17 patients fulfilled the inclusion criteria and agreed to participate [23], and just 12 completed all scans on all 3 scanning days. Owing to the low number of eligible patients, a crossover design was chosen to improve the power. In spite of these efforts, the low number of subjects limits the sensitivity of the study and potentially increases the risk of false-negative, as well as false-positive, results. Ideally, a multicenter study should be performed to clarify fully the extent of cerebrovascular deficits in patients with BMD and DMD.

\section{Conclusion}

In conclusion, patients with BMD who were treated for 4 weeks with sildenafil showed a significant increase in the somatosensory BOLD response compared with placebo without corresponding changes in the sensory-evoked potentials. No significant changes were detected in the remaining parameters pertaining to $\mathrm{CBF}$ and arterial diameter. These results indicate that there was a sildenafil-specific change in the neurovascular reactivity of patients with BMD, perhaps owing to altered dystrophin and nNOS function. 
Acknowledgments This work was supported by funding from LUCENS, the Gangsted Foundation, the Arup and Hvidt Foundation, the Jascha Foundation, the AP Møller Foundation, Capital Region Research Counsel, and the Lundbeck Foundation.

\section{Compliance with ethical standards}

Disclosure/Conflicts of Interest The authors declare no competing financial interests.

\section{References}

1. Bushby K, Finkel R, Birnkrant DJ, et al. Diagnosis and management of Duchenne muscular dystrophy, part 1: diagnosis, and pharmacological and psychosocial management. Lancet Neurol 2010;9:77-93.

2. Bushby KM, Goodship JA, Nicholson LV, Johnson MA, Haggerty ID, Gardner-Medwin D. Variability in clinical, genetic and protein abnormalities in manifesting carriers of Duchenne and Becker muscular dystrophy. Neuromuscul Disord 1993;3:57-64.

3. Emery AEH. The muscular dystrophies. Lancet 2002;359: 687-695.

4. Young HK, Barton BA, Waisbren S, et al. Cognitive and psychological profile of males with becker muscular dystrophy. J Child Neurol 2007;23:155-162.

5. Ricotti V, Mandy WP, Scoto M, et al. Neurodevelopmental, emotional, and behavioural problems in Duchenne muscular dystrophy in relation to underlying dystrophin gene mutations. Dev Med Child Neurol 2016;58:77-84.

6. Hoffman EP, Kunkel LM. Dystrophin abnormalities in Duchenne/ Becker muscular dystrophy. Neuron 1989;2:1019-1029.

7. Matsumura K, Campbell KP. Dystrophin-glycoprotein complex: its role in the molecular pathogenesis of muscular dystrophies. Muscle Nerve 1994;17:2-15.

8. Brenman JE, Chao DS, Xia H, Aldape K, Bredt DS. Nitric oxide synthase complexed with dystrophin and absent from skeletal muscle sarcolemma in Duchenne muscular dystrophy. Cell 1995;82:743-752.

9. Chang WJ, Iannaccone ST, Lau KS, et al. Neuronal nitric oxide synthase and dystrophin-deficient muscular dystrophy. Proc Natl Acad Sci U S A 1996;93:9142-9147.

10. Stamler JS, Meissner G. Physiology of nitric oxide in skeletal muscle. Physiol Rev 2001;81:209-237.

11. Szabadits E, Cserep C, Szonyi A, et al. NMDA Receptors in hippocampal GABAergic synapses and their role in nitric oxide signaling. J Neurosci 2011;31:5893-5904.

12. Costa ADT, Pierre SV, Cohen MV, Downey JM, Garlid KD. cGMP signalling in pre- and post-conditioning: the role of mitochondria. Cardiovasc Res 2007;77:344-352.

13. Torelli S, Brown SC, Jimenez-Mallebrera C, Feng L, Muntoni F, Sewry CA. Absence of neuronal nitric oxide synthase (nNOS) as a pathological marker for the diagnosis of Becker muscular dystrophy with rod domain deletions. Neuropathol Appl Neurobiol 2004;30: 540-545.

14. Rando TA. Role of nitric oxide in the pathogenesis of muscular dystrophies: a "two hit" hypothesis of the cause of muscle necrosis. Microsc Res Tech 2001;55:223-235.

15. Sander M, Chavoshan B, Harris SA, et al. Functional muscle ischemia in neuronal nitric oxide synthase-deficient skeletal muscle of children with Duchenne muscular dystrophy. Proc Natl Acad Sci U S A 2000;97:13818-13823.
16. Haenggi T, Fritschy JM. Role of dystrophin and utrophin for assembly and function of the dystrophin glycoprotein complex in non-muscle tissue. Cell Mol Life Sci 2006;63:1614-1631.

17. Waite A, Tinsley CL, Locke M, Blake DJ. The neurobiology of the dystrophin-associated glycoprotein complex. Ann Med 2009;41: 344-359.

18. Waite A, Brown SC, Blake DJ. The dystrophin-glycoprotein complex in brain development and disease. Trends Neurosci 2012;35:487-496.

19. Blake DJ, Hawkes R, Benson MA, Beesley PW. Different dystrophin-like complexes are expressed in neurons and glia. J Cell Biol 1999;147:645-658.

20. Adamo CM, Dai D-F, Percival JM, et al. Sildenafil reverses cardiac dysfunction in the mdx mouse model of Duchenne muscular dystrophy. Proc Natl Acad Sci U S A 2010;107:19079-19083.

21. Percival JM, Whitehead NP, Adams ME, Adamo CM, Beavo JA, Froehner SC. Sildenafil reduces respiratory muscle weakness and fibrosis in the mdx mouse model of Duchenne muscular dystrophy. J Pathol 2012;228:77-87.

22. De Arcangelis V, Strimpakos G, Gabanella F, et al. Pathways implicated in tadalafil amelioration of Duchenne muscular dystrophy. J Cell Physiol 2016;231:224-232.

23. Witting N, Kruuse C, Nyhuus B, et al. Effect of sildenafil on skeletal and cardiac muscle in Becker muscular dystrophy. Ann Neurol 2014;76:550-557.

24. Leung DG, Herzka DA, Thompson WR, et al. Sildenafil does not improve cardiomyopathy in Duchenne/Becker muscular dystrophy. Ann Neurol 2014;76:541-549.

25. Kruuse C, Thomsen LL, Jacobsen TB, Olesen J. The phosphodiesterase 5 inhibitor sildenafil has no effect on cerebral blood flow or blood velocity, but nevertheless induces headache in healthy subjects. J Cereb Blood Flow Metab 2002;22:1124-1131.

26. Rosengarten B, Schermuly RT, Voswinckel R, et al. Sildenafil improves dynamic vascular function in the brain: studies in patients with pulmonary hypertension. Cerebrovasc Dis 2006;21:194-200.

27. Doorenweerd N, Dumas EM, Ghariq E, et al. Reduced cerebral gray matter and altered white matter in boys with Duchenne muscular dystrophy. Neuromuscul Disord 2014;24:838-839.

28. Woolrich MW, Behrens TEJ, Smith SM. Constrained linear basis sets for HRF modelling using Variational Bayes. NeuroImage 2004;21:1748-1761.

29. de Koning PJH, Schaap JA, Janssen JP, Westenberg JJM, van der Geest RJ, Reiber JHC. Automated segmentation and analysis of vascular structures in magnetic resonance angiographic images. Magn Reson Med 2003;50:1189-1198.

30. Eickhoff SB, Stephan KE, Mohlberg H, et al. A new SPM toolbox for combining probabilistic cytoarchitectonic maps and functional imaging data. NeuroImage 2005;25:1325-1335.

31. Grefkes C, Geyer S, Schormann T, Roland P, Zilles K. Human somatosensory area 2 : observer-independent cytoarchitectonic mapping, interindividual variability, and population map. NeuroImage 2001;14:617-631.

32. Liu YJ, Juan CJ, Chen CY, et al. Are the local blood oxygen leveldependent (BOLD) signals caused by neural stimulation response dependent on global BOLD signals induced by hypercapnia in the functional MR imaging experiment? Experiments of long-duration hypercapnia and multilevel carbon dioxide concentration. AJNR Am J Neuroradiol 2007;28:1009-1014.

33. Lee JS, Pfund Z, Juhász C, et al. Altered regional brain glucose metabolism in Duchenne muscular dystrophy: a pet study. Muscle Nerve 2002;26:506-512.

34. Rae C, Scott RB, Thompson $\mathrm{CH}$, et al. Brain biochemistry in Duchenne muscular dystrophy: a $1 \mathrm{H}$ magnetic resonance and neuropsychological study. J Neurol Sci 1998;160:148-157.

35. Lv S-Y, Zou Q-H, Cui J-L, et al. Decreased gray matter concentration and local synchronization of spontaneous activity 
in the motor cortex in Duchenne muscular dystrophy. AJNR Am J Neuroradiol 2011;32:2196-2200.

36. Doorenweerd N, Straathof CS, Dumas EM, et al. Reduced cerebral gray matter and altered white matter in boys with Duchenne muscular dystrophy. Ann Neurol 2014;76:403-411.

37. Kobayashi YM, Rader EP, Crawford RW, et al. Sarcolemmalocalized nNOS is required to maintain activity after mild exercise. Nature 2008;456:511-515.

38. Martin EA, Barresi R, Byrne BJ, et al. Tadalafil alleviates muscle ischemia in patients with Becker muscular dystrophy. Sci Transl Med 2012;4:162ra155.

39. Nelson MD, Rader F, Tang X, et al. PDE5 inhibition alleviates functional muscle ischemia in boys with Duchenne muscular dystrophy. Neurology 2014;82:2085-2091.

40. Sekiguchi M. The role of dystrophin in the central nervous system: a mini review. Acta Myol 2005;24:93-97.

41. Uchino M, Teramoto H, Naoe H, Yoshioka K, Miike T, Ando M. Localisation and characterisation of dystrophin in the central nervous system of controls and patients with Duchenne muscular dystrophy. J Neurol Neurosurg Psychiatry 1994;57:426-429.

42. Cauli B, Tong X-K, Rancillac A, et al. Cortical GABA interneurons in neurovascular coupling: relays for subcortical vasoactive pathways. J Neurosci 2004;24:8940-8949.

43. Kruuse C, Hansen AE, Larsson HBW, Lauritzen M, Rostrup E. Cerebral haemodynamic response or excitability is not affected by sildenafil. J Cereb Blood Flow Metab 2009;29:830-839.
44. Gao F, Sugita M, Nukui H. Phosphodiesterase 5 inhibitor, zaprinast, selectively increases cerebral blood flow in the ischemic penumbra in the rat brain. Neurol Res 2005;27:638-643.

45. Arnavaz A, Aurich A, Weissenborn K, Hartmann U, Emrich HM, Schneider U. Effect of sildenafil (Viagra) on cerebral blood flow velocity: a pilot study. Psychiatry Res 2003;122:207-209.

46. Zhang ZG, Chopp M. Neurorestorative therapies for stroke: underlying mechanisms and translation to the clinic. Lancet Neurol 2009;8:491-500.

47. Thakur T, Sharma S, Kumar K, Deshmukh R, Sharma PL. Eur J Pharmacol 2013;714:515-521.

48. Chen X-M, Wang N-N, Zhang T-Y, Wang F, Wu C-F, Yang J-Y. Neuroprotection by Sildenafil: neuronal networks potentiation in acute experimental stroke. CNS Neurosci Ther 2013;20:40-49.

49. Chen BR, Kozberg MG, Bouchard MB, Shaik MA, Hillman EMC. A critical role for the vascular endothelium in functional neurovascular coupling in the brain. J Am Heart Assoc 2014;3: e000787.

50. Hillman EMC. Coupling mechanism and significance of the BOLD signal: a status report. Annu Rev Neurosci 2014;37:161-181.

51. Gómez-Vallejo V, Ugarte A, García-Barroso C, et al. Pharmacokinetic investigation of sildenafil using positron emission tomography and determination of its effect on cerebrospinal fluid cGMP levels. J Neurochem 2016;136:403-415.

52. Schultheiss D, Müller SV, Nager W, et al. Central effects of sildenafil (Viagra) on auditory selective attention and verbal recognition memory in humans: a study with event-related brain potentials. World J Urol 2001;19:46-50. 